\title{
Adaptação ao Ensino Superior, Estratégias de Aprendizagem e Motivação de Alunos EaD
}

\author{
Aline Ribeiro Bacan ${ }^{1}$ \\ ${ }^{1}$ Universidade São Francisco, SP, Brasil.
}

\author{
Gustavo Henrique Martins ${ }^{1}$ \\ ${ }^{1}$ Universidade São Francisco, SP, Brasil.
}

Acácia Aparecida Angeli dos Santos ${ }^{1}$

${ }^{1}$ Universidade São Francisco, SP, Brasil.

\begin{abstract}
Resumo: A Educação a Distância (EaD) é uma modalidade de ensino que tem crescido no Brasil, contudo pouco se sabe sobre o perfil adaptativo dos alunos EaD. Sendo assim, este estudo se propôs a avaliar alunos da modalidade a distância, considerando seu processo de adaptação ao ensino superior, o uso de estratégias de aprendizagem e de sua motivação para aprender. 324 universitários de cursos das áreas humanas, biológicas e exatas oferecidos integralmente a distância, de instituições de ensino pública e privada no interior do estado de São Paulo, responderam o questionário sociodemográfico, Questionário de Adaptação ao Ensino Superior (QAES/EaD), Escala de Estratégias de Aprendizagem para Universitários (EEA-U/EaD), Escala de Estratégias de Aprendizagem/EaD em contexto universitário híbrido (EEA-H) e Escala de Avaliação da Motivação para Aprendizagem (EMAPRE-U/EaD). Os resultados revelaram correlações positivas entre os construtos, tendo sido verificadas diferenças de gênero, idade e históricos de formação acadêmica. A partir deste estudo foi possível ampliar informações sobre o perfil do aluno de cursos EaD, possibilitando que as instituições de Ensino Superior possam oferecer-lhes ambientes virtuais mais adequados.
\end{abstract}

Palavras-chave: Educação a Distância, Avaliação Psicológica, Ensino Superior, Perfil do Estudante.

\section{Adaptation to Higher Education, Learning Strategies and Motivation of DL Students}

\begin{abstract}
Distance learning is a teaching and learning modality that has grown in Brazil, but little is known about the adaptive profile of distance learning students. Thus, this study evaluated distance learning students, considering their process of adaptation to higher education, the use of learning strategies and their motivation to learn. A total of 324 university students from courses in humanities, biological and exact sciences offered in full distance learning from public and private educational institutions in the countryside of the state of São Paulo participated. Participants answered a sociodemographic questionnaire, the Adaptation to Higher Education Questionnaire (QAES/EaD), Learning Strategies Scale for University Students (EEA-U/EaD), Learning Strategies Scale/EaD in a hybrid university context (EEA-H) and Scale of Motivation Evaluation for Learning (EMAPRE-U/EaD). The results revealed a positive correlation between the constructs, with differences observed according to gender, age of students and academic background. Based on this study, it was possible to further understand distance learning students, enabling higher education institutions to offer them more appropriate virtual environments.
\end{abstract}

Keywords: Distance Education, Psychological Evaluation, Higher Education, Student Profile. 


\title{
Adaptación a la Educación Superior, Estrategias de Aprendizaje y Motivación de Alumnos EaD
}

\begin{abstract}
Resumen: La Educación a Distancia (EaD) es una modalidad de enseñanza que ha crecido en Brasil, sin embargo, poco se sabe sobre el perfil adaptativo de los alumnos EaD. Ante esto, el presente estudio se propuso evaluar a alumnos de la modalidad a distancia, considerando su proceso de adaptación a la enseñanza superior, el uso de estrategias de aprendizaje y de su motivación para aprender. Participaron 324 universitarios de cursos de las áreas de humanas, biológicas y exactas ofrecidos íntegramente a distancia, de instituciones de enseñanza pública y privada en el interior del estado de São Paulo. Los participantes respondieron a cuestionarios sociodemográficos: Cuestionario de Adaptación a la Enseñanza Superior (QAES/EaD), Escala de Estrategias deAprendizajeparaUniversitarios (EEA-U/EaD), Escala deEstrategias deAprendizaje/ EaD en contexto universitario híbrido (EEA-H) y Escala de Evaluación de la Motivación para el Aprendizaje (EMAPRE-U/EaD). Los resultados revelaron correlaciones positivas entre los constructos y se verificaron diferencias de género, edad e históricos de formación académica. A partir de este estudio fue posible obtener la ampliación del conocimiento sobre el alumno de cursos EaD, lo que posibilita que las Instituciones de Enseñanza Superior puedan ofrecerles ambientes virtuales más adecuados.
\end{abstract}

Palabras clave: Educación a Distancia, Evaluación Psicológica, Enseñanza Superior, Perfil del Estudiante.

\section{Introdução}

A educação a distância, segundo o Decreto no 9.057, de 25 de maio de 2017, é uma modalidade de ensino que possibilita a mediação didático-pedagógica por meio de tecnologias de informação e comunicação, podendo fazer uso profissionais da educação e estudantes em lugares e tempos diversos (Brasil, 2017). Esta modalidade possibilita conhecer as modificações que se operam nos modos de percepção, nas possibilidades de interconectividade, nos conceitos de tempo e de espaço, nos diferentes estilos cognitivos, enfim, nos modos de existir, ser e estar dos envolvidos nesta forma de estudo (Saraiva, Pernigotti, Barcia, \& Lapolli, 2006). Os autores relatam que as habituais referências de tempo e espaço constitutivas dos sujeitos envolvidos nessa modalidade ocorrem sob nova ótica.

Segundo Gomes, Mota e Leonardo (2014) o êxito ou não de um estudante em curso superior na modalidade a distância está vinculado, entre outros fatores, ao seu perfil. Normalmente, alunos de cursos a distância são adultos, com filhos e ativos profissionalmente, além de buscarem uma aprendizagem mais orientada à prática, gostam de ter controle sobre seus atos e têm na EaD uma possibilidade de estudo
(Gomes et al., 2014; Guimarães, 2012). Tal tendência se confirma pelo Censo da Educação Superior de 2017 no que diz respeito aos cursos integralmente a distância. Em relação ao gênero, eles atendem ao público masculino e feminino em proporções relativamente equivalentes, com uma parcela levemente superior de participação feminina (62,6\%). Em termos de faixa etária, a média de idade dos alunos matriculados em cursos na modalidade a distância é de 32,3 anos $(D P=9,2)$ (Instituto Nacional de Estudos e Pesquisas Educacionais Anísio Teixeira, 2019).

Tem sido constatado que alunos matriculados em cursos na modalidade a distância apresentam dificuldades para organizar seus estudos e para assumir autonomamente a responsabilidade por sua aprendizagem (Acevedo, Torres, \& Tirado, 2015; Covalsky \& Mota, 2016; Garcia \& Carvalho, 2015). A respeito, Ramos (2013) refere a existência de um descompasso entre o perfil real e o ideal, que prevê características como as capacidades de exercer a autonomia e ser ativo nos processos de ensino e de aprendizagem.

Huang, Chandra, DePaolo e Simmons (2016) consideram que fatores ambientais, tais como o local que o aluno estuda (em casa, no trabalho e outros), 
a separação de espaço e tempo entre os envolvidos (aluno/professor/outros alunos) e as características demográficas do aluno (idade e gênero) afetam sua autonomia para estudar. No mesmo sentido, Acevedo et al. (2015) já referiam que a investigação das características desses alunos permite compreender como elas afetam o desempenho acadêmico e ampliam a chance de sucesso no ambiente virtual de aprendizagem.

Além da investigação sobre características sociodemográficas, como as encontradas nas pesquisas mencionadas, é necessário que sejam avaliados outros aspectos frequentemente associados à aprendizagem de alunos na educação presencial. Nesse sentido, acredita-se que a investigação de construtos psicológicos, que ajudem a compreender como ocorre a adaptação ao ensino superior na educação a distância, quais as estratégias de aprendizagem que seus alunos empregam e qual a sua motivação para aprender poderá fornecer elementos importantes sobre os alunos $\mathrm{EaD}$, permitindo ampliar o conhecimento sobre sua aprendizagem (Fleith; 2017; Oliveira, Boruchovitch, \& Santos, 2009; Oliveira, Santos, \& Inácio, 2017; Zenorini, Santos, \& Monteiro, 2011; Zerbini, Abbad, Mourão, \& Martins, 2015).

A adaptação do aluno à modalidade a distância é uma variável relevante para entender o comportamento do universitário no decorrer de sua formação, motivo pelo qual este é um dos construtos aqui abordados. É importante salientar que a adaptação ao ensino superior é um processo multifacetado, envolvendo diversos desafios intrapessoais, interpessoais e ambientais, que exigem o necessário desempenho de novos papéis para ajustamento ao novo contexto, bem como agir de forma proativa em relação à carreira escolhida (Almeida \& Soares, 2004; Astin, 1993).

O processo ensino-aprendizagem, tal como ocorre na educação a distância, também representa uma variável a ser investigada neste estudo, pois o processo não significa uma simplificação do realizado na modalidade presencial, uma vez que todo referencial teórico necessário à aquisição das competências e habilidades que promovam o desenvolvimento do indivíduo, o exercício da cidadania e a autonomia para continuar aprendendo é exatamente o mesmo para ambas as formas de ensino (Boruchovitch \& Santos, 2006). As autoras afirmam que as estratégias de aprendizagem têm se mostrado associadas ao sucesso da aprendizagem. O conceito assumido por elas é o de que são procedimentos e comportamentos escolhidos com intuito de facilitar a aquisição, armazenamento e aplicação do conhecimento. Assim, é essencial conhecer quais são as estratégias de aprendizagem empregadas pelos estudantes em cursos a distância.

Além das variáveis adaptação e estratégias de aprendizagem, a motivação dos universitários pode ser também considerada uma variável influente nos cursos superiores oferecidos na modalidade a distância. A motivação tem implicação direta com o envolvimento do aluno no processo de aprendizagem (Guimarães \& Boruchovitch, 2004). Uma forma bastante difundida de explicar a motivação para a aprendizagem é a assumida pela Teoria de Metas de Realização. Sob esta perspectiva, procura-se identificar os propósitos qualitativamente definidos pelo sujeito em situações de aprendizagem, nas quais necessita apresentar desempenho. Elas revelam o que o estudante estabelece para alcançar e se estruturam em três tipos: meta aprender (propósito de se envolver em atividades escolares para adquirir competências necessárias para o domínio da tarefa), meta performance aproximação (propósito de aparecer como melhor que os outros) e meta performance evitação (propósito de evitar situações que possa parecer pior que os outros) que, por sua vez, afetam a forma como se engajam nas tarefas (Bzuneck, 2004).

Tendo em vista os construtos adaptação, estratégias de aprendizagem e motivação da aprendizagem de universitários na educação a distância, algumas considerações podem ser feitas sobre os desafios envolvendo a dimensão humana e o modo de organização dos cursos superiores oferecidos na modalidade a distância. Com base nestas considerações, o objetivo deste trabalho foi avaliar alunos de cursos a distância, analisando o quanto os processos de adaptação ao ensino superior, o uso de estratégias de aprendizagem e sua motivação para aprender estão correlacionados. Ademais, os escores obtidos por eles foram comparados em razão de algumas características, tais como gênero, idade e histórico de formação acadêmica.

\section{Método}

\section{Participantes}

A amostra foi composta por 324 alunos matriculados em cursos superiores das áreas humanas, biológicas e exatas oferecidos integralmente na modalidade a distância, sendo 58,3\% do gênero feminino 
e faixa etária entre 18 e 60 anos $(M=28,9 ; D P=9,4)$. Os participantes foram provenientes de três instituições de ensino superior privadas e uma instituição pública. Todas as instituições de ensino se localizam no interior do estado de São Paulo.

\section{Instrumentos}

Os instrumentos Questionário de Adaptação ao Ensino Superior (QAES), Escala de Estratégias de Aprendizagem para Universitários (EEA-U) e Escala de Avaliação da Motivação para Aprendizagem (EMAPRE-U) foram utilizados e apresentam itens que foram adaptados pelos autores que consideraram o contexto da Educação a Distância. Também foi utilizada a Escala de Estratégias de Aprendizagem em contexto universitário híbrido (EEA-H). Cada uma delas será descrita em seguida.

Questionário Sociodemográfico: refere-se aos dados demográficos dos participantes. Assim foram identificadas variáveis como: gênero, idade, renda, estado civil, curso e semestre matriculado, históricos de formação acadêmica (anteriormente cursou ou não outra graduação) e domínio na utilização de tecnologia. Este questionário apresenta como objetivo identificar o perfil dos participantes que foram considerados como fatores relevantes na análise dos construtos do estudo.

Questionário de Adaptação ao Ensino Superior (QAES/EaD; Santos et al., 2017): o questionário é composto por 40 itens, divididos igualmente em cinco fatores sendo estes, Projeto de carreira, Adaptação social, Adaptação pessoal, Adaptação ao estudo e Adaptação institucional. Os itens se referem a vivências, opiniões e sentimentos em relação à adaptação acadêmica do aluno no Ensino Superior. A chave de resposta do instrumento é dada em uma escala Likert de cinco pontos, variando de "discordo totalmente (1)" a “concordo totalmente (5)". Na versão EaD foram adaptados 13 itens. Exemplos de itens adaptados: "As salas e os espaços físicos da minha Universidade agradam-me" (versão original), "As salas virtuais das disciplinas agradam-me" (versão EaD).

Escala de Estratégias de Aprendizagem para Universitários - EEA-U/EaD (Santos \& Boruchovitch, 2017): a escala é composta por 35 itens, respondidos em de escala tipo Likert, de quatro pontos, variando de "sempre (1)" a "nunca (4)". Os itens se referem à maneira como os alunos estudam ou se preparam para uma avaliação. $\mathrm{O}$ instrumento é formado por três fatores, sendo estes Autorregulação cognitiva e metacognitiva, Autorregulação dos recursos internos e contextuais e Autorregulação social. Exemplos de itens adaptados: "Rever as anotações feitas em sala de aula" (versão original), "Rever as anotações feitas durante as apresentações das videoaulas" (versão adaptada). Na versão $\mathrm{EaD}$ foram adaptados 14 itens e excluído um que não se enquadrou à modalidade de educação a distância.

Escala de Estratégias de Aprendizagem em contexto universitário híbrido - EEA-H (Martins \& Zerbini, 2014): a escala é composta por 29 itens, respondidos em forma de escala tipo Likert, de onze pontos, variando de "nunca (0)" a "sempre (10)". Os itens se referem à identificação e mensuração das estratégias de aprendizagem empregadas pelos alunos em cursos a distância e híbridos (semipresencial). A escala é composta por quatro fatores: Controle da emoção, Estratégias cognitivas, Estratégias autorregulatórias e Busca de ajuda interpessoal.

Escala de Avaliação da Motivação para Aprendizagem - EMAPRE-U/EaD (Santos \& Zenorini, 2017): a escala é composta por 28 itens, respondidos em forma de escala tipo Likert, de três pontos, variando de "concordo (1)" a "discordo (3)". Os itens se referem à motivação e às atitudes dos alunos em relação à aprendizagem. A escala é formada por três fatores, sendo estes Meta aprender, Meta performance e Meta performance-evitação. Exemplos de itens adaptados: "Não respondo aos questionamentos feitos pelo professor, por medo de falar alguma besteira" (versão original), "Não respondo aos questionamentos feitos pelo professor ou tutor, por medo de falar alguma besteira" (versão $\mathrm{EaD}$ ). Na versão $\mathrm{EaD}$ foram adaptados sete itens.

\section{Procedimento}

Após autorização das instituições e da aprovação do projeto pelo Comitê de Ética em Pesquisa da Universidade São Francisco (CAAE: 64829817.4.0000.5514), o propósito da pesquisa foi apresentado para os universitários de duas maneiras: por e-mail e apresentação individual. Àqueles que concordaram em participar foi disponibilizado, por e-mail e em vias impressas, o Termo de Consentimento Livre e Esclarecido (TCLE) com informações esclarecedoras acerca do estudo, o qual após lido, foi aceito por meio de autorização virtual ou assinatura nos casos dos participantes da coleta impressa. 
Conforme mencionado, os instrumentos foram aplicados em alguns casos on-line e em outros em formulários impressos. No caso de coleta presencial foram agendadas as datas específicas para a aplicação dos instrumentos nas instituições de ensino superior participantes, sendo reservado um local (sala de aula) para a realização da pesquisa. O tempo para preenchimento dos instrumentos (coleta on-line e presencial) foi de aproximadamente 30 minutos.

\section{Análise de dados}

Os dados foram analisados no programa estatístico SPSS v. 21. Inicialmente foram realizadas análises descritivas da amostra e das variáveis utilizadas. Em seguida foram realizadas correlações de Pearson entre os fatores dos instrumentos e interpretadas seguindo Cohen (1992) que considera os seguintes valores de $r$ 0,10 (fraca), 0,30 (moderada) $\mathrm{e}$ 0,50 (forte). As precisões dos fatores dos instrumentos foram analisadas por meio do coeficiente Alfa. Para verificar as diferenças de médias nos fatores dos instrumentos entre os grupos formados pelo gênero, foram utilizados o Teste $t$ de Student e o $d$ de Cohen. Ao passo que, para verificar as diferenças entre os grupos formados pela idade e históricos de formação acadêmica (cursou e concluiu, cursou e não concluiu ou não cursou outra graduação presencial ou a distância) foram utilizadas Análises de Variância (ANOVA) com provas post-hoc de Tukey. Os valores do $d$ de Cohen serão interpretados como $d \geq 0,2$ são pequenos, $d \geq 0,5$ são médios, e $d \geq 0,8$ são grandes (Cohen, 1992).

\section{Resultados}

Considerando os propósitos do estudo, primeiramente serão apresentadas as estatísticas descritivas, de precisão e os índices de correlação entre os instrumentos empregados. Assim, na Tabela 1 estão apresentadas as médias, os desvios padrões, as correlações e os valores Alfa de Cronbach de todos os fatores dos instrumentos usados para a avaliação dos construtos focalizados.

Tabela 1.

Estatísticas descritivas e correlações entre as variáveis.

\begin{tabular}{|c|c|c|c|c|c|c|c|c|c|c|c|c|c|c|c|c|}
\hline & $\mathrm{M}(\mathrm{DP})$ & 1 & 2 & 3 & 4 & 5 & 6 & 7 & 8 & 9 & 10 & 11 & 12 & 13 & 14 & 15 \\
\hline 1 & $3,82(0,63)$ & 0,84 & & & & & & & & & & & & & & \\
\hline 2 & $3,23(0,87)$ & $0,21^{* *}$ & 0,90 & & & & & & & & & & & & & \\
\hline 3 & $3,20(0,81)$ & $0,20^{* *}$ & 0,00 & 0,84 & & & & & & & & & & & & \\
\hline 4 & $3,56(0,54)$ & $0,48^{* *}$ & $0,39^{* *}$ & $0,16^{* *}$ & 0,74 & & & & & & & & & & & \\
\hline 5 & $3,37(0,65)$ & $0,45^{* *}$ & $0,37^{* *}$ & $0,26^{* *}$ & $0,43^{* *}$ & 0,81 & & & & & & & & & & \\
\hline 6 & $2,62(0,31)$ & $0,31^{* *}$ & $0,19^{* *}$ & $0,24^{* *}$ & $0,39^{* *}$ & $0,31^{* *}$ & 0,76 & & & & & & & & & \\
\hline 7 & $1,71(0,51)$ & 0,07 & 0,06 & $-0,08$ & 0,08 & 0,05 & 0,06 & 0,84 & & & & & & & & \\
\hline 8 & $1,42(0,48)$ & $-0,08$ & $-0,06$ & $-0,17^{* *}$ & $-0,12^{*}$ & $-0,02$ & $-0,22^{* *}$ & $0,41^{* *}$ & 0,85 & & & & & & & \\
\hline 9 & $3,07(0,44)$ & $0,25^{* *}$ & $0,28^{* *}$ & $0,12^{*}$ & $0,48^{* *}$ & $0,29^{* *}$ & $0,42^{* *}$ & $-0,01$ & $-0,21^{* *}$ & 0,88 & & & & & & \\
\hline 10 & $3,11(0,51)$ & $0,26^{* *}$ & $0,15^{* *}$ & $0,24^{* *}$ & $0,46^{* *}$ & $0,20^{* *}$ & $0,43^{* *}$ & 0,02 & $-0,23^{* *}$ & $0,65^{* *}$ & 0,76 & & & & & \\
\hline 11 & $2,54(0,78)$ & 0,09 & $0,55^{* *}$ & $-0,05$ & $0,25^{* *}$ & $0,18^{* *}$ & $0,21^{* *}$ & $0,13^{*}$ & $-0,02$ & $0,43^{* *}$ & $0,28^{* *}$ & 0,71 & & & & \\
\hline 12 & $6,44(1,82)$ & $0,22^{* *}$ & 0,04 & $0,31^{* *}$ & $0,22^{* *}$ & $0,12^{*}$ & $0,32^{* *}$ & 0,04 & $-0,13^{*}$ & $0,17^{* *}$ & $0,39^{* *}$ & $0,13^{*}$ & 0,79 & & & \\
\hline 13 & $7,19(1,44)$ & $0,32^{* *}$ & $0,18^{* *}$ & $0,19^{* *}$ & $0,46^{* *}$ & $0,22^{* *}$ & $0,43^{* *}$ & 0,02 & $-0,25^{* *}$ & $0,51^{* *}$ & $0,54^{* *}$ & $0,25^{* *}$ & $0,47^{* *}$ & 0,86 & & \\
\hline 14 & $5,25(2,73)$ & 0,09 & $0,46^{* *}$ & $-0,04$ & $0,21^{* *}$ & $0,13^{*}$ & $0,15^{* *}$ & $0,15^{* *}$ & 0,00 & $0,23^{* *}$ & $0,12^{*}$ & $0,60^{* *}$ & 0,10 & $0,17^{* *}$ & 0,79 & \\
\hline 15 & $6,70(1,57)$ & $0,31^{* *}$ & $0,29^{* *}$ & $0,17^{* *}$ & $0,41^{* *}$ & $0,29^{* *}$ & $0,40^{* *}$ & $0,15^{* *}$ & $-0,15^{* *}$ & $0,53^{* *}$ & $0,39^{* *}$ & $0,36^{* *}$ & $0,37^{* *}$ & $0,71^{* *}$ & $0,40^{* *}$ & 0,90 \\
\hline
\end{tabular}

Nota: Os valores na diagonal são Alfa de Cronbach.

Legenda: 1 = Projeto de carreira; 2 = Adaptação social; 3 = Adaptação pessoal; 4 = Adaptação ao estudo; $5=$ Adaptação institucional; 6 = Meta aprender; 7 = Meta performance-aproximação; 8 = Meta performance-evitação; $9=$ Autorregulação cognitiva e metacognitiva; 10 = Autorregulação dos recursos internos e contextuais; 11 = Autorregulação social; 12 = Controle da emoção; 13 = Estratégias autorregulatórias; 14 = Busca de ajuda interpessoal; 15 = Estratégias cognitivas.

${ }^{*}=p \leq 0,05 ;{ }^{* *}=p \leq 0,01$. 
Tal como observado na Tabela 1, dentre os fatores da QAES/EaD, que avaliaram a adaptação ao ensino superior, a única correlação positiva com magnitude forte observada ocorreu entre Adaptação social e Autorregulação social (EEA-U/EaD). O fator Projeto de carreira se correlacionou de forma positiva e com magnitude moderada aos fatores Meta aprender, Estratégias autorregulatórias e Estratégias cognitivas. O fator Adaptação social se correlacionou de forma positiva e com magnitude moderada também ao fator Busca de ajuda interpessoal. Os fatores Adaptação pessoal e Adaptação institucional se correlacionaram positivamente e com magnitudes moderadas apenas com um fator cada, sendo estes o fator Controle da emoção e Meta aprender, respectivamente. Por fim, o fator da QAES/EaD que apresentou mais correlações positivas e com magnitudes moderadas aos outros instrumentos foi a Adaptação ao estudo, o qual se correlacionou a Meta aprender, Autorregulação cognitiva e metacognitiva, Autorregulação dos recursos internos e contextuais, Estratégias autorregulatórias e Estratégias cognitivas. Por sua vez, em relação aos fatores da EMAPRE-U/EaD, que avaliou a motivação para aprender, apenas o fator Meta aprender apresentou correlações positivas e moderadas, as quais foram com Autorregulação cognitiva e metacognitiva, Autorregulação dos recursos internos e contextuais, Controle da emoção, Estratégias autorregulatórias e Estratégias cognitivas.

Na sequência, todos os fatores da EEA-U/EaD e EEA-H apresentaram correlações significativas e positivas entre si, com magnitudes variando de fracas a fortes. O fator Autorregulação cognitiva e metacognitiva se correlacionou fortemente aos fatores Estratégias autorregulatórias e Estratégias cognitivas. Por sua vez, o fator Autorregulação dos recursos internos e contextuais apresentou correlações com magnitude forte com Estratégias autorregulatórias, e com magnitudes moderadas a Controle da emoção e Estratégias cognitivas. O fator Autorregulação social apresentou correlação forte com Busca de ajuda interpessoal e correlação moderada com Estratégias cognitivas.

A fim de verificar se houve diferença nas pontuações dos fatores dos instrumentos de acordo como o gênero dos estudantes, foi realizado um Teste t, assim como calculados os valores do $d$ de Cohen, para se verificar o tamanho do efeito dessa diferença. A frequência encontrada entre os dois grupos foi de 189 (58,3\%) do gênero feminino e $135(41,7 \%)$ do gênero masculino. Na Tabela 2 são apresentadas apenas as diferenças que foram significativas para $p<0,05$.

Os dados da Tabela 2 mostram que um fator de cada instrumento avaliado apresentou diferença nas médias dos grupos formados pelo gênero. No caso da Adaptação pessoal, Meta performance-aproximação e Controle da emoção os estudantes do gênero masculino obtiveram maior média que as estudantes do gênero feminino, sendo observado o contrário em relação ao fator Autorregulação cognitiva, no qual as mulheres obtiveram maior média. Os tamanhos de efeito foram todos pequenos, exceto para o fator Controle da emoção que indicou um tamanho de efeito moderado, favorecendo os homens.

A seguir, na Tabela 3, serão apresentadas as diferenças de médias nos fatores dos instrumentos entre os grupos formados pelas idades dos universitários. Para a realização desta análise, as idades foram categorizadas em três faixas, considerando a distribuição dos participantes da amostra. A primeira foi formada pelos alunos com idades de 18 a 22 anos $(n=107 ; 33 \%)$, a segunda agrupou alunos com idades entre 23 a 31 anos $(n=105 ; 32,4 \%)$ e a terceira e última faixa etária foi formada pelos que tinham idades de 32 a 60 anos $(n=112 ; 34,6 \%)$.

Tabela 2.

Teste $\mathrm{t}$ de Student para as variáveis utilizadas entre os grupos formados pelo gênero.

\begin{tabular}{lccccc}
\hline \multicolumn{1}{c}{ Variáveis } & Média feminino $(D P)$ & Média masculino $(D P)$ & $T$ & $p$ & $d$ \\
\hline Adaptação pessoal & $3,12(0,83)$ & $3,31(0,77)$ & $-2,21$ & 0,03 & 0,24 \\
Meta aproximação & $1,64(0,46)$ & $1,80(0,57)$ & $-2,83$ & 0,01 & 0,31 \\
Autorregulação cognitiva & $3,12(0,44)$ & $2,99(0,44)$ & 2,54 & 0,01 & 0,30 \\
Controle da emoção & $6,05(1,81)$ & $6,97(1,71)$ & $-4,61$ & 0,01 & 0,52 \\
\hline
\end{tabular}

Nota: Foram apresentados apenas os casos com $p<0,05$. 
Tabela 3.

ANOVA e prova de Tukey para as variáveis avaliadas entre os grupos formado pelas idades.

\begin{tabular}{|c|c|c|c|c|c|}
\hline \multirow[b]{2}{*}{ Variáveis } & \multirow[b]{2}{*}{$F$} & \multirow[b]{2}{*}{$p$} & \multirow[b]{2}{*}{ Idade } & \multicolumn{2}{|c|}{ Subconjuntos } \\
\hline & & & & 1 & 2 \\
\hline \multirow[t]{3}{*}{ Adaptação social } & 13,11 & 0,01 & 18 a 22 & 2,95 & \\
\hline & & & 23 a 31 & 3,29 & \\
\hline & & & 32 a 60 & & 3,52 \\
\hline \multirow[t]{3}{*}{ Adaptação pessoal } & 4,72 & 0,01 & 18 a 22 & 3,00 & \\
\hline & & & 32 a 60 & & 3,30 \\
\hline & & & 23 a 31 & & 3,30 \\
\hline \multirow[t]{3}{*}{ Adaptação ao estudo } & 4,16 & 0,02 & 18 a 22 & 3,43 & \\
\hline & & & 23 a 31 & 3,60 & 3,60 \\
\hline & & & 32 a 60 & & 3,63 \\
\hline \multirow[t]{3}{*}{ Meta aprender } & 6,97 & 0,01 & 18 a 22 & 2,55 & \\
\hline & & & 23 a 31 & 2,61 & 2,61 \\
\hline & & & 32 a 60 & & 2,70 \\
\hline \multirow[t]{3}{*}{ Meta performance-evitação } & 3,89 & 0,02 & 32 a 60 & 1,34 & \\
\hline & & & 23 a 31 & 1,41 & 1,41 \\
\hline & & & 18 a 22 & & 1,52 \\
\hline \multirow[t]{3}{*}{ Autorregulação cognitiva e metacog. } & 4,37 & 0,01 & 18 a 22 & 2,99 & \\
\hline & & & 23 a 31 & 3,04 & 3,04 \\
\hline & & & 32 a 60 & & 3,16 \\
\hline \multirow[t]{3}{*}{ Autorregulação dos recursos internos } & 3,30 & 0,04 & 18 a 22 & 3,03 & \\
\hline & & & 23 a 31 & 3,09 & 3,09 \\
\hline & & & 32 a 60 & & 3,20 \\
\hline \multirow[t]{3}{*}{ Autorregulação social } & 4,29 & 0,01 & 23 a 31 & 2,45 & \\
\hline & & & 18 a 22 & 2,45 & \\
\hline & & & 32 a 60 & & 2,71 \\
\hline \multirow[t]{3}{*}{ Busca de ajuda interpessoal } & 4,98 & 0,01 & 18 a 22 & 4,87 & \\
\hline & & & 23 a 31 & 4,94 & \\
\hline & & & 32 a 60 & & 5,90 \\
\hline
\end{tabular}

Nota: Foram apresentados apenas os casos com $p<0,05$.

${ }^{*} \mathrm{gl}=2$.

Os dados da Tabela 3 permitiram verificar que, pelo menos em um fator de cada instrumento, houve diferença entre as médias em relação aos grupos formados pela idade. As diferenças significativas ocorreram predominantemente entre o(os) grupo(os) formado(os) pelos estudantes com mais idade (32 a
60 anos e/ou 23 a 31 anos), que se diferenciou(aram) do grupo formado pelos estudantes de menos idade (18 a 22 anos), sendo que os mais velhos obtiveram maiores pontuações nos fatores. No caso específico do fator Meta performance-evitação é desejável que os estudantes apresentem médias mais baixas, visto 
que as médias mais altas apontam para maior frequência de comportamentos evitativos, de não envolvimento. Como nos outros fatores, o grupo formado pelos estudantes mais velhos (32 a 60 anos) obteve as menores médias em relação aos demais, indicando que costumam evitar menos as atividades acadêmicas. Os fatores que apresentaram diferenças de médias foram Adaptação social, Adaptação pessoal, Adaptação ao estudo, Meta aprender, Meta performance-evitação, Autorregulação cognitiva e metacognitiva, Autorregulação dos recursos internos e contextuais, Autorregulação social e Busca de ajuda interpessoal.

Para a realização da última análise, os participantes foram divididos em grupos referentes a seus históricos de formação acadêmica. A esse respeito, 214 estudantes disseram que não haviam cursado outra graduação, 52 disseram que cursaram outra graduação presencial e não a concluíram, 56 disseram que cursaram outra graduação presencial e concluíram, e dois estudantes responderam que haviam cursado outra graduação a distância, sendo que um concluiu o curso e outro não. Pelo fato, de apenas dois estudantes formarem o grupo que cursaram outra graduação a distância, esses dois casos foram excluídos. Sendo assim, os grupos para essa análise foram: não cursaram outa graduação $(n=214$; $66,5 \%)$, cursaram e não concluíram ( $n=52 ; 16,1 \%)$ e cursaram e concluíram ( $n=56 ; 17,4 \%)$. A seguir, na Tabela 4, estão descritas a ANOVA com a prova de Tukey para os grupos formados pelos estudantes que cursaram ou não outra graduação.

Os dados da Tabela 4 indicaram que os fatores Meta aprender e Meta performance-evitação da EMAPRE-U/EaD foram os únicos dentre todos os fatores testados que apresentaram diferenças nas médias para aqueles que cursaram ou não outra graduação anteriormente. No caso da Meta aprender, os estudantes que cursaram e concluíram outra graduação presencial apresentaram maiores médias que os estudantes que cursaram e não concluíram outra graduação. Por sua vez, no fator Meta performance-evitação os estudantes que cursaram e não concluíram outra graduação, e os que não cursaram outra graduação obtiveram maiores pontuações em comparação aos estudantes que cursaram e concluíram outra graduação presencial.

Tabela 4.

ANOVA e prova de Tukey para as variáveis avaliadas entre os grupos formados pelo histórico de formação acadêmica.

\begin{tabular}{|c|c|c|c|c|c|}
\hline \multirow[b]{2}{*}{ Variáveis } & \multirow[b]{2}{*}{$F$} & \multirow[b]{2}{*}{$p$} & \multirow[b]{2}{*}{ Cursar outra graduação } & \multicolumn{2}{|c|}{ Subconjuntos } \\
\hline & & & & 1 & 2 \\
\hline \multirow[t]{3}{*}{ Meta aprender } & 3,80 & 0,02 & Cursaram e não concluíram & 2,57 & \\
\hline & & & Não cursaram & 2,60 & 2,60 \\
\hline & & & Cursaram e concluíram & & 2,72 \\
\hline \multirow[t]{3}{*}{ Meta performance-evitação } & 4,78 & 0,01 & Cursaram e concluíram & 1,25 & \\
\hline & & & Não cursaram & & 1,45 \\
\hline & & & Cursaram e não concluíram & & 1,51 \\
\hline
\end{tabular}

Nota: Foram apresentados apenas os casos com $\mathrm{p}<0,05$.

$* \mathrm{gl}=2$.

\section{Discussão}

As dificuldades encontradas no contexto universitário são de natureza diversa. Dentre elas estão as questões pessoais dos alunos, as exigências acadêmicas que devem ser desempenhadas com eficiência e a adaptação a um novo ambiente, fatores estes que influenciam tanto o desempenho acadêmico como o desenvolvimento psicossocial dos estudantes. Isto pode prejudicar a motivação para aprender dos alunos (Cunha \& Carrilho, 2005). Nesse sentido, a adaptação dos alunos de ensino superior parece estar associada tanto a suas metas de aprendizagem como às estratégias de aprendizagem que utiliza. 
Conforme apresentado, foi constatada relação predominantemente positiva entre os construtos, com exceção da Meta performance-evitação que, devido à sua característica, era esperado teoricamente que houvesse correlações significativas, negativas e com magnitudes fracas com as demais. É importante salientar que os estudantes orientados pela meta performance-evitação veem as situações como uma ameaça e evitam se expor, com receio do fracasso, a qualquer situação que possa mostrar a sua incapacidade (Santos, Mognon, Alcará, \& Lemos, 2011).

Outro ponto que merece destaque é a correlação positiva com magnitudes moderada e forte entre a Adaptação social, avaliada pelo QAES/EaD, e Busca de ajuda interpessoal e Autorregulação social, avaliadas pela EEA-H e EEA-U/EaD respectivamente. A Adaptação social está vinculada à integração social do estudante no estabelecimento de relações, sendo esperada a existência de correlação positiva com tais estratégias de aprendizagem sociais. Isso se deve ao fato de que o estabelecimento de relações com os envolvidos (alunos, professores e instituição de ensino) facilita o processo de aprender a aprender, que implica ter autonomia e responsabilidade com o ato de estudar e ainda regular e planejar suas tarefas (Fernandes \& Frison, 2015). Ela se revela ainda pela busca de ajuda interpessoal e pela procura ativa, por iniciativa do próprio aluno, pela ajuda de outras pessoas (tutores ou pares) para tirar dúvidas. Segundo Warr e Allan (1998), tais estratégias comportamentais representam um comportamento proativo do indivíduo de buscar auxílio, ao invés de obter informações do material do curso. Por sua vez, Carmo e Franco (2019) trazem importantes considerações sobre as competências necessárias aos tutores, que são diversas e complexas, de ordem didático-pedagógica, tecnológica, linguística e social, para que sua atuação seja relevante para o alcance do sucesso no curso a distância.

O Projeto de carreira do QAES/EaD também mostrou correlação positiva com as Estratégias autorregulatórias, Estratégias cognitivas da EEA-H e ainda com a Meta aprender da EMAPRE-U/EaD. O aluno inserido no mercado de trabalho na área de conhecimento do curso que realiza percebe com mais clareza a importância da capacitação e educação na sua vida profissional. A Estratégia cognitiva é aquela que conduz o aluno a refletir sobre seu próprio pensamento para realizar uma atividade com sucesso e alcançar seu objetivo de aprendizado (Zimmerman, 2008).
Neste sentido, a estratégia de aprendizagem, seja ela metacognitiva, que leva o aluno a pensar, ou autorregulatória, que estimula o aluno a aprender, quando utilizadas simultaneamente resultam em satisfação com o curso (Barbosa, Carvalho, Soares Neto, \& Costa, 2014; Silva, Leal, Pereira, \& Oliveira Neto, 2015). Segundo Zerbini e Abbad (2010), as características individuais podem exercer interferência mais significativa nos resultados de ações educacionais a distância do que naqueles de natureza presencial, uma vez que a aprendizagem efetiva está, nesse primeiro caso, subordinada muito mais ao empenho empreendido pelo próprio sujeito (autorregulação da aprendizagem) do que aos recursos educacionais disponíveis.

Os resultados obtidos sobre a correlação positiva entre Projeto de carreira e Meta aprender são relatados também na teoria, a qual define a Meta aprender como envolvendo a busca por tarefas desafiadoras, pelo prazer que estas despertam, e relaciona-se com a motivação intrínseca, características existentes em alunos que almejam nos cursos de graduação a distância a possibilidade de crescimento profissional, por exemplo. Bzuneck (2004) ressalta que a Meta aprender diz respeito a resultados positivos, ao esforço e à persistência na busca de conhecimentos e no aprimoramento das habilidades, sendo, desta forma, propulsora de crescimento intelectual.

Observou-se ainda nos resultados a correlação positiva entre a Adaptação pessoal do QAES/EaD com Controle da emoção da EEA-H. Estas correlações revelam que a adaptação do aluno $\mathrm{EaD}$ tem relação direta com características intrínsecas do aluno. Sendo assim, isso indica que o autocontrole emocional utilizado pelo aluno para controlar os próprios processos psicológicos de aprendizagem favorece sua adaptação pessoal em situações de atenção, aquisição, memorização e transferência do conhecimento (Martins \& Zerbini, 2014).

A universidade tem sido considerada espaço de aprendizagem e de socialização. Assim, o ambiente universitário favorece o desenvolvimento cognitivo e afetivo (Guimarães \& Boruchovitch, 2004). A correlação aqui encontrada entre Adaptação institucional e Meta aprender corroboram a ideia de que para os alunos $\mathrm{EaD}$, mesmo não estando fisicamente em uma sala de aula nem vivenciando de forma presencial o ambiente universitário, consideram a adaptação institucional como importante aspecto para o processo de adaptação do aluno ao Ensino Superior na modalidade a distância. Assim sendo, o ambiente virtual de aprendizagem deve 
oferecer ao aluno $\mathrm{EaD}$ instrumentos de socialização tais como: videoaula, chat, fórum para dúvidas e para avisos da instituição, bem como outros meios didáticos de comunicação e interação com os envolvidos no processo de ensino e aprendizagem, para que o aluno permaneça motivado a aprender.

A respeito das correlações observadas entre os fatores dos instrumentos que medem estratégias de aprendizagem (EEA-U/EaD e EEA-H), foram constatadas correlações positivas e com magnitudes fortes entre os fatores correspondentes de ambos os instrumentos (Martins \& Zerbini, 2014; Santos \& Boruchovitch, 2017). O fator Autorregulação cognitiva e metacognitiva da EEA-U/EaD mostrou-se correlacionado a Estratégias autorregulatórias e Estratégias cognitivas da EEA-H. Por sua vez, a maior correlação da Autorregulação dos recursos internos e contextuais foi observada com Estratégias autorregulatórias. Por fim, o fator interpessoal da EEA-U/EaD (Autorregulação social) se correlacionou fortemente a Busca de ajuda interpessoal, da forma como era esperado. Sendo assim, estes resultados congruentes apontam para iniciais evidências de validade baseada na relação com outras variáveis para a versão EaD da EEA-U.

Foram exploradas, ainda, neste estudo eventuais diferenças nas pontuações dos fatores dos instrumentos de acordo como o gênero dos estudantes, idade e históricos de formação acadêmica. Os resultados encontrados em cada uma das variáveis são discutidos a seguir. A primeira análise estudada foi relativa ao gênero dos participantes, a qual apontou que os homens pontuaram mais em relação ao fator Controle da emoção da EEA-H com tamanho de efeito moderado. Desta forma este resultado sugere que homens costumam conseguir manter a calma mais facilmente frente às dificuldades enfrentadas durante o curso em comparação às mulheres. Este resultado se assemelha com o que é constantemente relatado por pesquisas realizadas em diferentes culturas a respeito do traço neuroticismo do Big Five, o qual os homens comumente apresentam menores níveis, o que significa que eles costumam ser mais estáveis emocionalmente (Schmitt et al., 2016).

$\mathrm{Na}$ observação das demais diferenças relativas ao gênero dos participantes, na EMAPRE-U/EaD os resultados foram superiores na Meta performance-aproximação para os homens. $\mathrm{O}$ aluno caracterizado por esta meta é entendido como aquele que sente necessidade de se destacar entre os colegas, mostrando-se mais inteligente, sem que o seu foco primordial esteja na aprendizagem efetiva (Bzuneck, 2004). Nesse sentido, verifica-se que a Meta performance-aproximação favorece indiretamente o desempenho acadêmico em termos de notas, evidenciando que o estudante do gênero masculino apresenta características que estimulam positivamente os seus estudos quando oferecidos na modalidade a distância.

Desta forma, os resultados obtidos revelam que estudantes do gênero masculino organizam melhor o tempo e o lugar de estudo, possuindo mais autodisciplina que as estudantes mulheres. Achados estes que se divergem dos encontrados no estudo de Santos, Mognon, Lima e Cunha (2011), as quais a partir de uma amostra de universitários de cursos presenciais, indicaram que as estudantes do gênero feminino pontuaram mais em Meta performance-aproximação em comparação aos estudantes do sexo masculino. Esses achados divergentes sugerem que novas pesquisas com objetivos parecidos devem ser realizadas a fim de se obter mais dados para que seja possível haver consenso sobre tais diferenças de médias encontradas.

Conforme apresentado nos resultados, os participantes foram divididos por idade, sendo obtidas diferenças que indicam que os estudantes com mais idade (32 a 60 anos), se diferenciaram do grupo formado pelos estudantes de menos idade (18 a 22 anos), sendo que os mais velhos obtiveram maiores pontuações nos fatores Autorregulação cognitiva e metacognitiva, Autorregulação dos recursos internos e contextuais e Autorregulação social da EEA-U/EaD e Busca de ajuda interpessoal da EEA-H. Resultados semelhantes foram apresentados no estudo de Marini e Boruchovitch (2014), no qual as autoras identificaram que estudantes mais velhos possuem maior capacidade de autorregular a aprendizagem.

Sendo ainda que os estudantes da faixa etária entre 32 a 60 anos pontuaram mais em Adaptação social, Adaptação pessoal e Adaptação ao estudo do QAES/EaD e Meta aprender da EMAPRE-U/EaD. Ao passo que os mais velhos também pontuaram menos no fator Meta performance-evitação, isto retrata que o aluno mais velho não tem medo de se expor no ambiente virtual favorecendo sua adaptação, aprendizagem e a motivação no decorrer do curso. A respeito, o Censo do Ensino Superior de 2017 (Instituto Nacional de Estudos e Pesquisas Educacionais Anísio Teixeira, 2019) indicou que apenas $25 \%$ dos alunos 
matriculados em cursos EaD têm idades superiores a 38 anos, observa-se que essa modalidade ainda tem potencial para crescer entre alunos de faixas etárias mais avançadas, seja por um possível desejo desses alunos de mudarem de carreira, seja por interesses e necessidades pessoais.

Na última análise realizada com a variável de agrupamento históricos de formação acadêmica (cursou e concluiu, cursou e não concluiu ou não cursou outra graduação presencial ou a distância) os resultados revelaram que os estudantes que já cursaram outra graduação presencial anteriormente e a concluíram possuem maior experiência e também maior motivação, seja para estudar ou para se engajar em atividades de participação de fóruns, chats tira-dúvidas e atividades de avaliação, a fim de se obter domínio sobre o conteúdo. Por outro lado, os estudantes que responderam ter cursado outra graduação presencial sem a concluirem pontuaram mais no fator meta performance-evitação. Indicando que os alunos que não cursaram ou que já possuem uma graduação realizada presencialmente apresentaram escores mais baixos em alguns aspectos prejudiciais ao estudo, deixando assim de evitar atividades que possam demonstrar sua competência.

\section{Considerações finais}

O crescimento da educação a distância no Brasil vem intensificando a necessidade de conhecer o perfil do aluno virtual, que passa a ser um sujeito ativo da sua própria aprendizagem. $\mathrm{Na} \mathrm{EaD} \mathrm{o} \mathrm{aproveitamento}$ do curso depende prioritariamente da postura do aluno, que é estimulado a ter uma postura ativa. Sem horário fixo de aula e sem a figura do professor presente o tempo todo, ele tem que assumir o controle de seus horários e criar um esquema de autocontrole para estudar e cumprir as tarefas. Contudo, a estrutura tecnológica oferecida pela instituição, bem como o preparo dos tutores poderão, em muito, contribuir para que o curso seja concluído com sucesso.

Diante da dinâmica de aprendizagem promovida pelo ensino a distância, os resultados apresentados indicaram que os alunos que possuem comportamentos adaptativos favoráveis a modalidade a distância são homens, na faixa etária entre 32 a 60 anos e que já tenham cursado e concluído anteriormente outra graduação na modalidade presencial. Observou-se na pesquisa que alunos com este perfil apresentam entendimento maior das demandas da aprendizagem a distância e maior comprometimento com o processo educacional que auxilia na manutenção do envolvimento e do engajamento de seus estudos. Cabe ressaltar que as mulheres apresentam maiores prejuízos em sua empregabilidade e produtividade comparada aos homens, devido à dupla jornada (trabalho-casa) que assumem (Beltrame \& Donelli, 2012; Silva, 2019), podendo isso impactar também na adaptação das mulheres ao ensino a distância. No entanto, saber disso importa criar alternativas para que os alunos que não têm este perfil possam também alcançar sucesso acadêmico e obter a formação desejada em cursos a distância.

O conhecimento do perfil do aluno virtual amplia as possibilidades de desenvolvimento e aperfeiçoamento das metodologias de ensino nesta modalidade favorecendo a adaptação e a motivação deste aluno. Apesar dos benefícios deste estudo, algumas limitações puderam ser identificadas, em especial o fato de os instrumentos terem sido planejados para serem respondidos na plataforma on-line e terem sido quase inteiramente aplicados em lápis e papel, em razão de dificuldades estruturais para o acesso aos instrumentos de pesquisa. $\mathrm{O}$ uso do instrumento on-line precisa ser repensado para que possa de fato ser utilizado de forma compatível àquela em que os alunos estudam. Os resultados aqui alcançados abrem possibilidades de aplicação prática. Profissionais envolvidos com a educação a distância poderão auxiliar no redirecionamento da prática de gestores e de professores que ministram cursos na modalidade a distância, que necessitam ficar mais atentos a sinais de desadaptação ao curso. A identificação de sinais de desmotivação e a dificuldade já identificada para estudar sozinho deverão ser clara e diretamente abordadas pelos docentes, que poderão adotar uma postura mais compreensiva a respeito delas e fornecerem sugestões práticas que facilitem ao aluno alternativas para lidar com tais dificuldades.

De modo geral pode-se verificar que os objetivos propostos nesta pesquisa foram alcançados satisfatoriamente, conforme apresentados e discutidos neste artigo. Alguns resultados necessitam ser mais bem explorados por novos estudos que aprofundem as questões levantadas, sobretudo em relação a todos os atores envolvidos no processo de ensino a distância (alunos, tutores/professores e instituição de ensino). Em termos de agenda futura, fica a sugestão de novas pesquisas, com amostras ampliadas e diversificadas, 
que possibilite a realização de análise de evidências de validade com base na estrutura interna dos itens dos instrumentos QAES/EaD, EEA-U/EaD e EMAPRE-U/EaD para a versão EaD. Com amostras mais amplas, poderão ser realizadas análises fatoriais confirmatórias, visto que os modelos teóricos e a estrutura fatorial dos instrumentos aqui empregados já estão bem definidas. Sem dúvida, a realização de estudos longitudinais permitirão acompanhar o aluno ingressante no curso a distância até sua conclusão, avaliando mudanças comportamentais que possam ocorrer, mostrando no dia a dia dos alunos e em diferentes disciplinas como ocorrem as dimensão do processo de adaptação, como desenvolvem estratégias de aprendizagem mais adequadas e como estabelecem as metas de realização no contexto $\mathrm{EaD}$.

\section{Referências}

Acevedo, D., Torres, J. D., \& Tirado, D. F. (2015). Análisis de los hábitos de estudio y motivación para el aprendizaje a distancia en alumnos de ingeniería de sistemas de la universidad de Cartagena (Colombia). Formación Universitaria, 8(5), 59-66. http://dx.doi.org/10.4067/S0718-50062015000500007

Almeida, L. S., \& Soares, A. P. (2004). Os estudantes universitários: Sucesso escolar e desenvolvimento psicossocial. In E. Mercuri \& S. A. J. Polydoro (Orgs.), Estudante universitário: Características e experiências de formação (pp. 15-40). Taubaté, SP: Cabral Editora.

Astin, A. (1993). What matters in college? San Francisco: Jossey-Bass.

Barbosa, S. I., Carvalho, D. L., Soares Neto, J. B., \& Costa, F. J. (2014). Uma análise dos condicionantes da satisfação, da dedicação e do desempenho de estudantes de cursos de administração. Administração: Ensino e Pesquisa, 15(2), 323-349. http://www.spell.org.br/documentos/ver/31586/uma-analise-dos-condicionantes-da-satisfacao--da -dedicacao-e-do-desempenho-de-estudantes-de-cursos-de-administracao/i/pt-br

Beltrame, G. R., \& Donelli, T. M. S. (2012). Maternidade e carreira: desafios frente à conciliação de papéis. Aletheia, (38-39), 206-217. http://pepsic.bvsalud.org/pdf/aletheia/n38-39/n38-39a17.pdf

Boruchovitch, E., \& Santos, A. A. A. (2006). Estratégias de aprendizagem: conceituação e avaliação. In A. P. P. Noronha, F. F. Sisto, \& A. A. A. Santos (Eds.), Facetas do fazer em avaliação psicológica (pp. 107-124). São Paulo, SP: Vetor.

Brasil. (2017). Decreto ${ }^{\circ}$ 9.057, de 25 de maio de 2017. Brasília, DF: Presidência da República. http:/ /www.planalto. gov.br/ccivil_03/_ato2015-2018/2017/decreto/d9057.htm

Bzuneck, J. A. (2004). A motivação do aluno orientado a metas de realização. In E. Boruchovitch \& J. A. Bzuneck (Orgs), A motivação do aluno: Contribuições da psicologia contemporânea (pp. 58-77). Petrópolis, RJ: Vozes.

Carmo, R. O. S., \& Franco, A. P. (2019). Da docência presencial à docência online: aprendizagens de professores universitários na educação a distância. Educação em Revista, 35, e210399.

Cohen, J. (1992). A power primer. Psychological Bulletin, 112(1), 155-159. http://www2.psych.ubc.ca/ schaller/ 528Readings/Cohen1992.pdf

Covalsky, C. M., \& Mota, J. C. (2016). Limites e possibilidades de estudantes na educação a distância (EaD). Revista da UNIFEBE, 1(18), 75-87. http://periodicos.unifebe.edu.br/index.php/revistaeletronicadaunifebe/article/ viewFile/399/216

Cunha, S. M., \& Carrilho, D. M. (2005). O processo de adaptação ao ensino superior e o rendimento acadêmico. Psicologia Escolar e Educacional, 9(2), 215-224. http://dx.doi.org/10.1590/S1413-85572005000200004

Fernandes, V. R., \& Frison, L. M. B. (2015). Estratégias de aprendizagem autorregulatória no ensino superior: escrita de um artigo científico. Psicologia da Educação, (41), 37-49. http:/ /dx.doi.org/10.5935/2175-3520.20150013

Fleith, D. S. (2017). Criatividade, motivação para aprender, ambiente familiar e superdotação: um estudo comparativo. Psicologia: Teoria e Pesquisa, 32(50), 1-9. http://dx.doi.org/10.1590/0102-3772e32ne211

Garcia, V. L., \& Carvalho, P. M., Jr. (2015). Educação à distância (EAD), conceitos e reflexões. Medicina, 48(3), 209-213. http://dx.doi.org/10.11606/issn.2176-7262.v48i3p209-213

Gomes, S. G. S., Mota, J. B., \& Leonardo, E. S. (2014). Reflexão sobre o perfil do aluno como determinante para a motivação e aprendizagem em cursos de EaD. Cadernos de Educação, Tecnologia e Sociedade, 7, 355-363. http://dx.doi.org/10.14571/ cets.v7.355-363 
Guimarães, L. S. R. (2012). O aluno e a sala de aula virtual. In F. M. Litto \& M. Formiga, (Org.), Educação a distância: O estado da arte. São Paulo, SP: Pearson.

Guimarães, S. E. R. \& Boruchovitch, E. (2004). O estilo motivacional do professor e a motivação intrínseca dos estudantes: uma perspectiva da teoria da autodeterminação. Psicologia, Reflexão e Crítica, 17(2), 143-150. http://www.scielo.br/pdf/prc/v17n2/22466.pdf

Huang, X., Chandra, A., \& DePaolo, C. A. (2016), Understanding transactional distance in web-based learning environments: An empirical study. British Journal of Educational Technology, 47(4), 734-737. http://dx.doi.org/10.1111/bjet.12263

Instituto Nacional de Estudos e Pesquisas Educacionais Anísio Teixeira. (2019). Resumo técnico do Censo da Educação Superior 2017. http://portal.inep.gov.br/web/guest/censo-da-educacao-superior

Marini, J. A. S., \& Boruchovitch, E. (2014). Estratégias de aprendizagem de alunos brasileiros do ensino superior: Considerações sobre adaptação, sucesso acadêmico e aprendizagem autorregulada. Revista Eletrônica de Psicologia, Educação e Saúde, 4(1), 102-126. https://revistaepsi.com/wp-content/uploads/artigos/2014/Ano4Volumel-Artigo5.pdf

Martins, L. B., \& Zerbini, T. (2014). Escala de estratégias de aprendizagem: Evidências de validade em contexto universitário híbrido. Psico-USF, 19(2), 317-328. http://dx.doi.org/10.1590/1413-82712014019002007

Martins, L. B., \& Zerbini, T. (2016). Fatores influentes no desempenho acadêmico de universitários em ações educacionais a distância. Estudos de Psicologia, 21(3), 317-327. http://dx.doi.org/10.5935/1678-4669.20160030

Oliveira, K. L., Boruchovitch, E., \& Santos, A. A. A. (2009). Estratégias de aprendizagem e desempenho acadêmico: Evidências de validade. Psicologia: Teoria e Pesquisa, 25(4), 531-536. http://www.scielo.br/pdf/ptp/v25n4/a08v25n4

Oliveira, K. L., Santos, A. A. A., \& Inácio, A. L. M. (2017). Estratégias de aprendizagem no ensino médio brasileiro: Análise exploratória dos resultados. Revista de Estudios e Investigación em Psicología y Educación, Extr.(1), 337-341. http://dx.doi.org/10.17979/reipe.2017.0.01.3041

Pascarella, E. T., \& Terenzini, P. T. (2005). How college affects students: A third decade of research. San Francisco: Jossey-Bass.

Ramos, K. R. (2013). Perfil dos alunos de licenciatura a distância e aspectos que contribuem para aprendizagem. Revista Reflexão e Ação, 21(n. esp), 199-220. http://dx.doi.org/10.17058/rea.v21i2.2847

Santos, A. A. A., \& Boruchovitch. E. (2017). Escala de avaliação de estratégias de aprendizagem - adaptada para universitários de cursos a distância. Campinas, SP: Universidade São Francisco; Unicamp.

Santos, A. A. A., Mognon, J. F., Alcará, R. A., \& Lemos, T. H. (2011). Motivação para aprender: evidência de validade convergente entre duas medidas. Aletheia, 35(36), 36-50. http://pepsic.bvsalud.org/ scielo.php?script=sci_arttext\&pid=S1413-03942011000200004

Santos, A. A. A., Mognon, J. F., Lima, T. H., \& Cunha, N. B. (2011). A relação entre vida acadêmica e a motivação para aprender em universitários. Psicologia Escolar e Educacional, 15(2), 283-290. http://www.redalyc.org/ $\mathrm{html} / 2823 / 282321817010 /$

Santos, A. A. A., Noronha, A. P. P., Zanon, C., Araújo, A. M, Ferreira, J. A., \& Almeida, L. S. (2017). Questionário de Adaptação ao Ensino Superior (QAES) - adaptado para cursos a distância no Brasil. Relatório não publicado. Campinas, SP: Universidade São Francisco.

Santos, A. A. A., \& Zenorini, R. P. (2017). Escala de motivação para aprendizagem - versão EaD. Relatório não publicado. Campinas, SP: Universidade São Francisco.

Saraiva. L. M., Pernigotti, J. M., Barcia, R. M., \& Lapolli, E. M. (2006). Tensões que afetam os espaços de educação a distância. Psicologia em Estudo, 11(3), 483-491. http://dx.doi.org/10.1590/S1413-73722006000300004

Schmitt, D. P., Long, A. E., McPhearson, A., O’Brien, K., Remmert, B., \& Shah, S. H. (2016). Personality and gender differences in global perspective. International Journal of Psychology, 52(51), 1-12. http://dx.doi.org/10.1002/ ijop. 12265

Silva, D. M., Leal, E. A., Pereira, J. M., \& Oliveira Neto, J. D. (2015). Estilos de aprendizagem e desempenho acadêmico na Educação a Distância: uma investigação em cursos de especialização. Revista Brasileira de Gestão de Negócios, 17(57), 1300-1316. http:// dx.doi.org/10.7819/rbgn.v17i57.1852

Silva, L. L. T. (2019). Mulheres e o mundo do trabalho: a infindável dupla jornada feminina. Revista Eletrônica Interações Sociais, 3(1), 120-131. https://periodicos.furg.br/reis/article/view/9171 
Warr, P., \& Allan, C. (1998). Learning strategies and occupational training. Internacional Review of Industrial and OrganizationalPsychology,13,83-121.https://books.google.com.br/books?id=MkVBx4n5Y6EC\&printsec=frontcover\& $\mathrm{hl}=\mathrm{pt}-\mathrm{BR} \# \mathrm{v}=$ onepage $\& \mathrm{q} \& \mathrm{f}=$ false

Zenorini,R.P.C.,Santos,A.A.A.,\&Monteiro,R.M.(2011).Motivaçãoparaaprender:relaçãocomodesempenhodeestudantes. Paidéia, 21(49), 157-164.https://www.researchgate.net/profile/Acacia_Santos2/publication/262591144_ Motivation_for_learning_The_relation_with_students_performance/links/0a85e53c7f7b56dfb6000000/ Motivation-for-learning-The-relation-with-students-performance.pdf

Zerbini, T., \& Abbad, G. (2010). Aprendizagem induzida pela instrução em contexto de organizações e trabalho: uma análise crítica da literatura. Cadernos de Psicologia Social do Trabalho, 13(2), 177-193. http://dx.doi.org/10.11606/ issn.1981-0490.v13i2p177-193

Zerbini, T., Abbad, G., Mourão, L., \& Martins, L. B. (2015). Estratégias de aprendizagem em curso corporativo a distância: Como estudam os trabalhadores? Psicologia: Ciência e Profissão, 35(4), 1024-1041. http://dx.doi.org/10.1590/1982-3703000312014

Zimmerman, B. J. (2008). Investigating self-regulation and motivation: Historical background, methodological developments and future prospects. American Educational Research Journal, 45(1), $166-183$. http:// dx.doi.org/10.3102/0002831207312909

\section{Aline Ribeiro Bacan}

Professora e mestre pela Universidade São Francisco, Campinas - SP. Brasil.

E-mail: alinerbacan@yahoo.com.br

(i) http://orcid.org/0000-0002-4282-4820

\section{Gustavo Henrique Martins}

Doutorando pelo Programa de Pós-Graduação em Psicologia da Universidade São Francisco, Campinas - SP. Brasil. E-mail: gustavoh.martins95@gmail.com

(iD) http://orcid.org/0000-0002-5125-2553

Acácia Aparecida Angeli dos Santos

Professora doutora pelo Programa Pós-Graduação em Psicologia da Universidade São Francisco, Campinas - SP. Brasil.

E-mail: acacia.angeli@gmail.com

(D) http://orcid.org/0000-0003-2544-8891

Endereço para envio de correspondência:

Universidade São Francisco, Departamento de Pós-Graduação Stricto Sensu em Psicologia. Rua Waldemar César da Silveira, 105, Jardim Cura D’ars. CEP: 13045-510. Campinas - SP. Brasil.

Recebido $13 / 08 / 2018$

Aceito 03/07/2020

Received 13/08/2018

Approved 03/07/2020

Recibido 13/08/2018

Aceptado 03/07/2020 
Como citar: Bacan, A. R., Martins, G. H., Santos, A. A. A. (2020). Adaptação ao Ensino Superior, Estratégias de Aprendizagem e Motivação de Alunos EAD. Psicologia: Ciência e Profissão, 40, 1-15.

https://doi.org/10.1590/1982-3703003211509

How to cite: Bacan, A. R., Martins, G. H., Santos, A. A. A. (2020). Adaptation to Higher Education, Learning Strategies and Motivation of DE Students. Psicologia: Ciência e Profissão, 40, 1-15.

https://doi.org/10.1590/1982-3703003211509

Cómo citar: Bacan, A. R., Martins, G. H., Santos, A. A. A. (2020). Adaptación a la Educación Superior, Estrategias de Aprendizaje y Motivación de Alumnos EAD. Psicologia: Ciência e Profissão, 40, 1-15. https://doi.org/10.1590/1982-3703003211509 